\title{
Plenitude: Aplicativo mobile para melhorar a qualidade de vida de pessoas com transtornos depressivos e ansiedade
}

\author{
Geraldo Castro Carvalho Júnior ${ }^{1}$, Anderson Soares Costa ${ }^{1}$ \\ ${ }^{1}$ Núcleo de Tecnologia da Informação (NTI) - Universidade CEUMA \\ São Luís - MA - Brasil \\ juniorcastrz@gmail.com, anderson.lsdi.ufma.br
}

\begin{abstract}
The work describes the development of a mobile application on Android and IOS platforms aimed at people with depressive and anxiety disorders, disorders common in the 21st century and that can even lead to suicide. Emphasis was placed on methodological procedures: requirements gathering and analysis, as well as the use of agile methodologies. The work aims to increase the quality of life of people through the use of a mobile application capable of showing motivational phrases, recording and monitoring events and user status daily, informing through publications, videos and podcasts. health professionals, more specifically psychologists and therapists, through a partnership.
\end{abstract}

Resumo. $O$ trabalho descreve o desenvolvimento de um aplicativo mobile nas plataformas Android e IOS voltado para pessoas com transtornos depressivos e ansiedade, transtornos comuns no século XXI e que podem levar até mesmo ao suicídio. Deu-se ênfase aos procedimentos metodológicos: levantamento e análise de requisitos, bem como a utilização de metodologias ágeis. O trabalho visa aumentar a qualidade de vida das pessoas através do uso de um aplicativo mobile capaz de mostrar frases motivacionais, registrar e acompanhar eventos e estado do usuário diariamente, informar através de publicações, vídeos e podcasts, para isso, teve-se respaldo de profissionais da área da saúde, mais precisamente psicólogos e terapeutas, através de uma parceria.

\section{Introdução}

$\mathrm{O}$ número de pessoas com acesso à internet tem crescido abruptamente nos últimos anos. O Brasil é o $4^{\circ}$ Lugar no ranking de número de usuários de internet com pouco mais de 139 milhões de pessoas usufruindo de tal tecnologia, este número representa $70,5 \%$ da população do país, segundo dados da União Internacional de Telecomunicações (UIT, 2017) ${ }^{1}$.

O número de pessoas com transtornos de ansiedade e depressão, por sua vez, também têm crescido. De acordo com dados divulgados pela Organização Mundial da Saúde (OMS), em 2017, a porcentagem da população que sofre com transtornos depressivos é de 5,8\%, quase o dobro da média mundial. Em relação apenas à ansiedade, esse mal acomete cerca de $9,3 \%$ da população brasileira, tornando o país o número um no ranking de países com mais pessoas ansiosas ${ }^{2}$. Pessoas com tais transtornos geralmente se isolam e recebem duras críticas por parte de amigos e familiares, isso faz com que elas fiquem relutantes em buscar informações sobre o que sentem, enquanto isso, o estado físico e mental tende a piorar, podendo levar até mesmo ao suicídio. 
O real motivo de números tão agressivos é desconhecido, porém, ao traçar um paralelo entre estes dois temas, é possível perceber que utilizando a tecnologia, pode-se reduzir a incidência e até mesmo prevenir que alguns novos casos da doença aconteçam. Com isso, o presente trabalho tem como objetivo desenvolver um aplicativo que auxilie as pessoas na busca por informação sobre saúde mental, bem como melhorar a qualidade de vida, fazer com que se sintam à vontade e entendam um pouco mais sobre si mesmas.

\section{Fundamentação Teórica}

\subsection{Depressão}

De acordo com um levantamento do Ministério da Saúde (2015), a depressão é uma doença grave e que tem se tornado cada vez mais comum na vida das pessoas, ela afeta negativamente a forma como o indivíduo pensa, se comporta e age, independentemente de estar em grupo ou sozinho. ${ }^{3}$

Com sintomas considerados inofensivos por muitos, a depressão pode variar de leve à grave num curto espaço de tempo, podendo levar até mesmo ao suicídio. Manifestações recorrentes de tristeza ou humor deprimido, baixa autoestima, perda de energia e fadiga extrema, alterações de apetite e perda no interesse de atividades que antes eram consideradas prazerosas não devem ser ignorados ou tratados como bobagem, pois são os principais indícios da presença da doença, afirma o site especializado em psicologia Vittude (2016). ${ }^{4}$

Segundo dados divulgados pela Organização Mundial da Saúde (OMS, 2017), estima-se que $5,8 \%$ da população brasileira convive com o problema de depressão, número este que está acima da média mundial, e ao levar em conta que pessoas que têm depressão geralmente não procuram ajuda médica, este número pode ser assustadoramente maior. ${ }^{2}$

Não há um consenso dos pesquisadores sobre as reais causas da depressão, visto que ela pode surgir tanto sem motivo algum quanto ser desencadeado por algum evento importante como falência, perda de emprego, morte de um ente querido ou divórcio. Tais acontecimentos são chamados gatilhos. Segundo o Ministério da Saúde (2018), a doença não está necessariamente ligada aos acontecimentos, pois a vida de alguém pode estar em perfeita condição e mesmo assim a pessoa pode apresentar a doença em qualquer um dos graus. Já na tristeza, o indivíduo tem consciência de que está triste e sabe o motivo que gerou tal sentimento, logo, não se deve confundir tristeza com depressão. ${ }^{2}$

\subsection{Sistemas de informação na saúde}

$\mathrm{O}$ advento da internet, juntamente com telefones inteligentes trouxe uma enorme facilidade na busca por informações. Hoje, praticamente tudo que um indivíduo precisa saber está a alguns toques de distância. Segundo o Relatório Estado de Serviços Móveis (ESM, 2018), os brasileiros passaram mais de três horas por dia utilizando o celular. O número de aplicativos móveis nas lojas virtuais também tem crescido em todos os sistemas operacionais. O que geralmente acontece é que as pessoas não utilizam essa praticidade a seu favor, seja porque não tem conhecimento da doença ou das ferramentas.

Os benefícios da utilização da tecnologia no diagnóstico e acompanhamento de usuários ou pacientes com problemas de saúde mental e qualidade vida são inúmeros, vão desde o acesso à informação fácil e gratuita ao diagnóstico precoce de alguma doença 
mais séria e até mesmo melhora significativa na qualidade de vida das pessoas. "Essa tecnologia moderna, criada pelo homem, à serviço do homem, tem contribuído em larga escala para a solução de problemas antes insolúveis, e pode reverter em melhores condições de vida e saúde para as pessoas", diz pesquisa publicada na SciELO (2012). ${ }^{5}$

\section{Metodologia}

Para o desenvolvimento dos componentes de softwares da infraestrutura foram utilizados princípios dos chamados Métodos Ágeis de Desenvolvimento de Software que preveem a construção de software de forma incremental, interativa e adaptativa. Esta escolha se deve à necessidade de utilizar um processo ágil de desenvolvimento, que seja razoavelmente documentado e fácil de ser gerenciado. Sempre que possível, o desenvolvimento incremental do software foi baseado em técnicas de refatoração.

O software de desenvolvimento do aplicativo foi o Android Studio, linguagem de programação dart e framework Flutter, que é um kit de desenvolvimento interface usuário desenvolvido pela Google e lançado em 2017 .Já dentro do código, foram implementados gráficos, interação com outras funcionalidades do aparelho e conexão com o banco de dados em nuvem Firebase, já que é referência em segurança, agilidade e praticidade, além de ser um banco de dados não relacional, que será de extrema importância para futuras funcionalidades do projeto.

\section{Análise e projeto}

\subsection{Análise de requisitos}

O quadro 1 representa os requisitos funcionais, que segundo Vazquez, Carlos; Simões, Guilherme (2016) "define uma função de um sistema de software ou seu componente". 6

Quadro 1 - Requisitos Funcionais APP Usuário

\begin{tabular}{|c|c|}
\hline \multicolumn{2}{|l|}{ RF - APP Usuário (Principal) } \\
\hline $\mathbf{R F}$ & Descrição \\
\hline [RF001]. Gerenciar usuário & $\begin{array}{l}\text { O sistema deverá permitir que o usuário se cadastre, faça login e } \\
\text { alteração de dados. }\end{array}$ \\
\hline [RF002]. Mostrar números de ajuda & $\begin{array}{l}\text { O sistema deverá mostrar números de emergência como Centro de } \\
\text { Valorização da Vida (CVV), Bombeiros, Polícia. }\end{array}$ \\
\hline [RF003]. Mostrar multimídia & $\begin{array}{l}\text { O sistema deverá ter uma aba para multimídia em que haverá } 3 \text { sub } \\
\text { abas, sendo elas Publicações, Vídeos e Podcasts }\end{array}$ \\
\hline [RF004]. Mostrar Frases & O sistema deverá conter uma aba para mostrar frases motivacionais \\
\hline [RF005]. Favoritar Itens & $\begin{array}{l}\text { O sistema deverá permitir o usuário de favoritar suas publicações, } \\
\text { frases ou vídeos. }\end{array}$ \\
\hline $\begin{array}{l}\text { [RF006]. Analisar dados de estado } \\
\text { mental do usuário }\end{array}$ & $\begin{array}{l}\text { O sistema deverá mostrar os dados dos últimos } 7 \text { ou } 30 \text { dias do } \\
\text { estado do usuário. }\end{array}$ \\
\hline
\end{tabular}

Fonte: $\mathrm{O}$ autor 
O quadro 2 representa os requisitos funcionais mais importantes do aplicativo de inserção de dados que poderá ser usado apenas pelos profissionais da área, afim de tornar o app principal mais adequado com informações precisas de profissionais formados. Neste quadro são expressos apenas os requisitos que ficarão acessíveis ao profissional.

Quadro 2 - Requisitos Funcionais APP Secundário

\begin{tabular}{|l|l|}
\hline \multicolumn{2}{|l|}{ RF - APP Profissional (Secundário) } \\
\hline RF & Descrição \\
\hline [RF001]. Analisar e aceitar frases & $\begin{array}{l}\text { O sistema deverá permitir que o usuário (clínica) analise as frases } \\
\text { enviadas pelos usuários comuns do app principal, podendo libera-las } \\
\text { ou não. }\end{array}$ \\
\hline [RF002]. Fazer publicações & $\begin{array}{l}\text { O sistema deverá permitir que o usuário (clínica) faça suas } \\
\text { publicações com fotos, títulos, descrição e tags. }\end{array}$ \\
\hline [RF003]. Analisar diário de usuário & $\begin{array}{l}\text { O sistema deverá permitir que o usuário (clínica) analise os dados de } \\
\text { estado do usuário do app principal (paciente) afim de ter dados mais } \\
\text { precisos sobre como essa pessoa tem se sentido ao longo do tempo. }\end{array}$ \\
\hline [RF004]. Postar vídeos & O sistema deverá permitir que o usuário (clínica) envie vídeos. \\
\hline
\end{tabular}

Fonte: $\mathrm{O}$ autor

O quadro 3 representa os requisitos não funcionais de ambos os aplicativos. Requisitos não funcionais são, segundo Vazquez, Carlos; Simões, Guilherme (2016) "requisitos relacionados ao uso da aplicação em termos de desempenho, usabilidade, confiabilidade, segurança, disponibilidade, manutenção e tecnologias envolvidas". 6

Quadro 3 - Requisitos não Funcionais

\begin{tabular}{|l|l|}
\hline \multicolumn{2}{|l|}{ RNF - APP Usuário e Profissional } \\
\hline RNF & Descrição \\
\hline [RNF001]. Rápido e responsivo & $\begin{array}{l}\text { O sistema deverá ser rápido em todas as plataformas e o tempo de } \\
\text { resposta deve ser mais otimizado possível }\end{array}$ \\
\hline [RNF002]. Adaptável & O sistema deverá mostrar consistente ao tipo de tela \\
\hline [RNF003]. Banco de dados na nuvem & $\begin{array}{l}\text { O sistema deverá ter um banco de dados hospedado na nuvem e } \\
\text { online 24h por dia 7 dias por semana. }\end{array}$ \\
\hline
\end{tabular}

Fonte: $\mathrm{O}$ autor

O quadro 4 representa as regras de negócio também de ambos os aplicativos, regras de negócio especificam as particularidades do sistema.

Quadro 4 - Regras de Negócio

\begin{tabular}{|l|l|}
\hline \multicolumn{2}{|l|}{ RN - APP Usuário e Profissional } \\
\hline RN & Descrição \\
\hline [RN001]. Login com email e google & O sistema deverá possibilitar o login via email ou conta google. \\
\hline [RN002]. Usuário logado & $\begin{array}{l}\text { Apenas usuários logados poderão favoritar itens e alterar dados do } \\
\text { perfil. }\end{array}$ \\
\hline
\end{tabular}




\begin{tabular}{|l|l|} 
[RN003]. Acesso ilimitado & $\begin{array}{l}\text { O usuário terá acesso ilimitado e gratuito a todas as funcionalidades } \\
\text { do aplicativo. }\end{array}$ \\
\hline [RN004]. Clínicas & $\begin{array}{l}\text { Clínicas credenciadas poderão fazer publicações, consulta de diário } \\
\text { de pacientes, envio e autorização de frases e marcação de consulta. }\end{array}$ \\
\hline
\end{tabular}

Fonte: $\mathrm{O}$ autor

\subsection{Projeto}

A Figura 1 mostra a tela de login e cadastro do aplicativo, ambas em funcionamento e salvando os usuários cadastrados diretamente no Firebase, banco de dados em nuvem da Google.

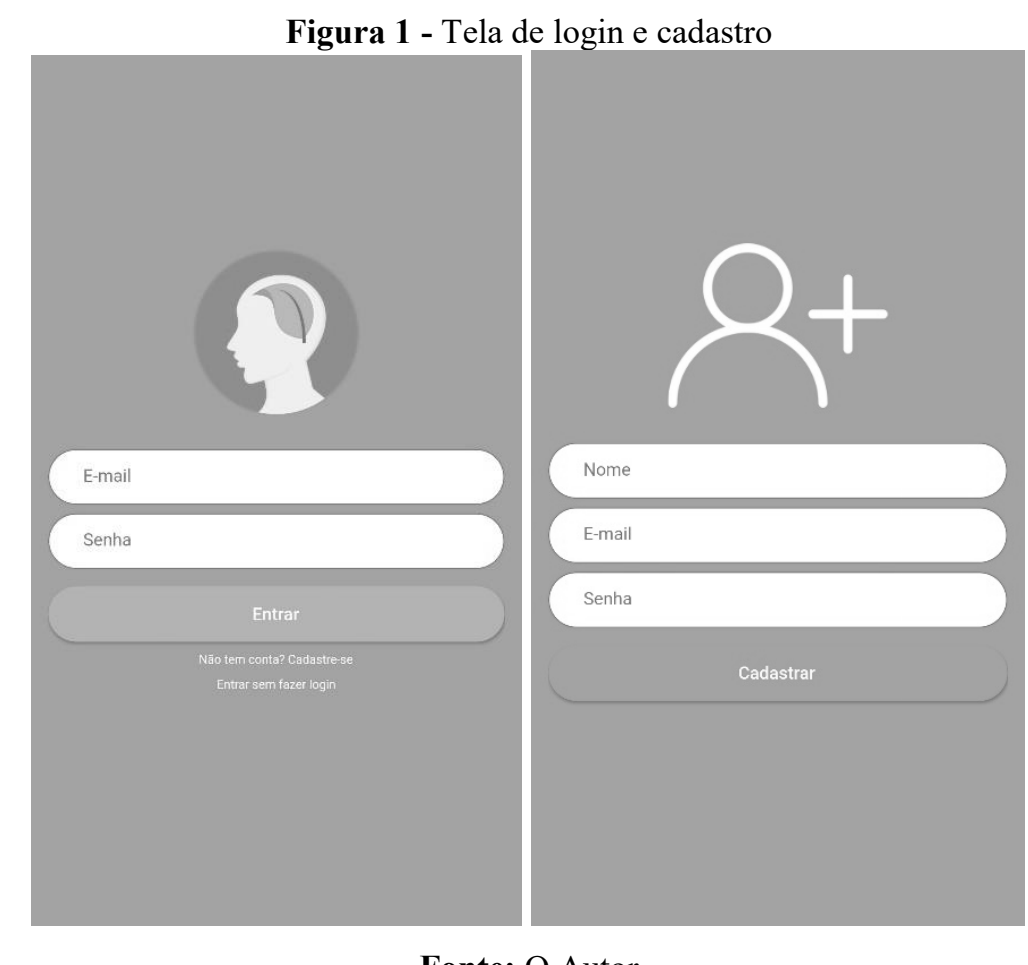

Fonte: O Autor

A Figura 2 mostra a tela inicial, que é mostrada após o usuário realizar o login, ela é composta por uma barra de navegação inferior, a qual existem quatro itens, que redirecionam o usuário cada um para uma das seguintes abas: frases, diário, multimídia e menu. A "Home" do aplicativo já é o primeiro item, nele é possível gerar uma frase aleatória do banco de dados, assim como a opção de torna-la favorita.

Figura 2 - Página inicial (Frases) 


\section{Fraser}

"Clique abaixo para gerar uma frase"

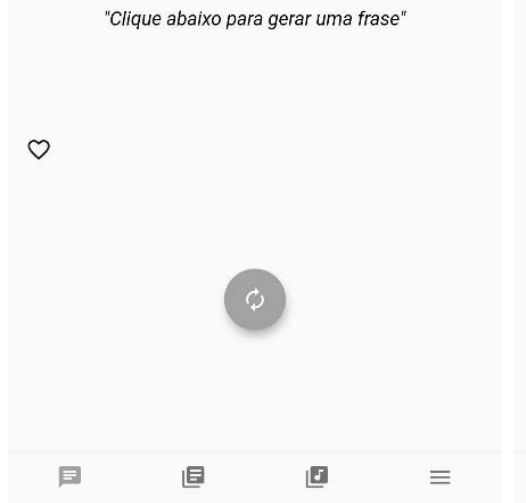

\section{Frases}

"Os problemas são oportunidades para se mostrar o que sabe."

O
- Duke Ellington

Fonte: O Autor

A Figura 3 mostra a aba "Diário" e a sub aba "Hoje", em que o usuário pode fazer uma espécie de diário com o adicional de poder expressar como se sente através dos emoticons de faces. Cada face corresponde a um número, tais números são tratados pelo sistema afim de mostrar com maior precisão estatísticas e análise de como o usuário tem se sentido ao longo do tempo, funcionalidade essa que também facilita a vida do profissional da saúde que dar suporte a esse usuário/paciente. Já na sub aba "Calendário", o usuário pode escolher um dia específico para editar ou simplesmente verificar como se sentiu e o que escreveu no dia selecionado.

Figura 3 - Página diário e calendário

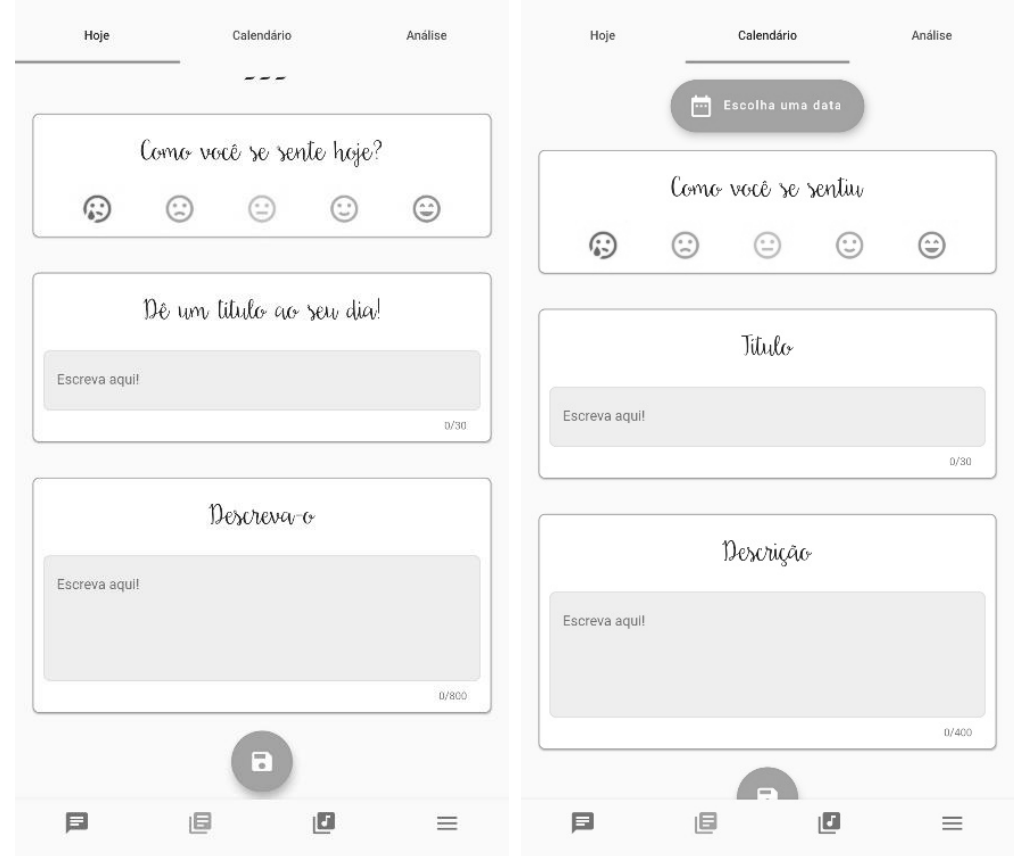




\section{Fonte: O Autor}

A Figura 4 mostra a aba publicações, em que também é possível pesquisar por título. Essas publicações são feitas apenas pelos profissionais da área e são de cunho informativo.

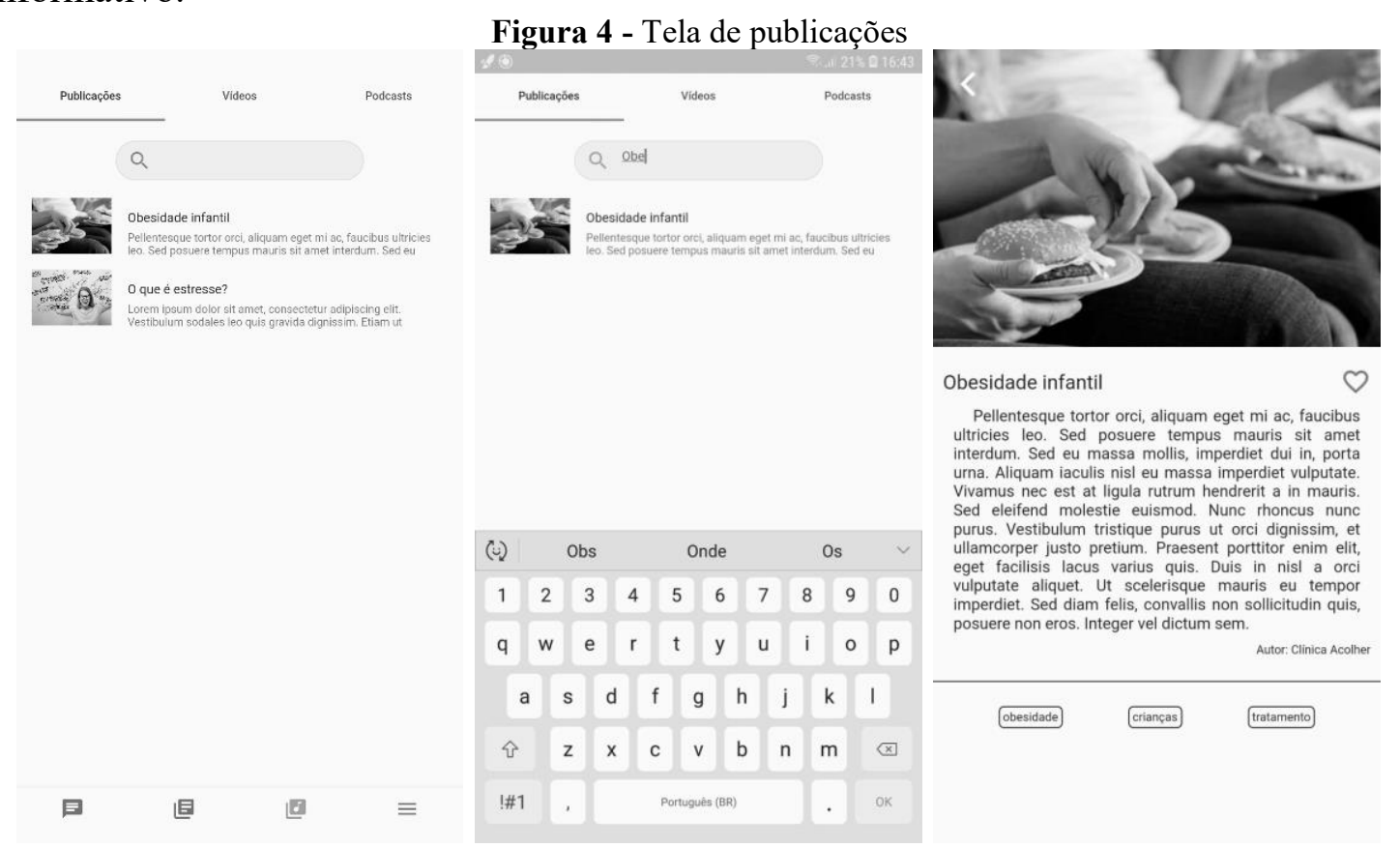

Fonte: O Autor

A Figura 5 mostra a aba menu, o item de ajuda e alguns botões que redirecionam o usuário para o aplicativo nativo de ligações do seu aparelho, independentemente de marca ou modelo.

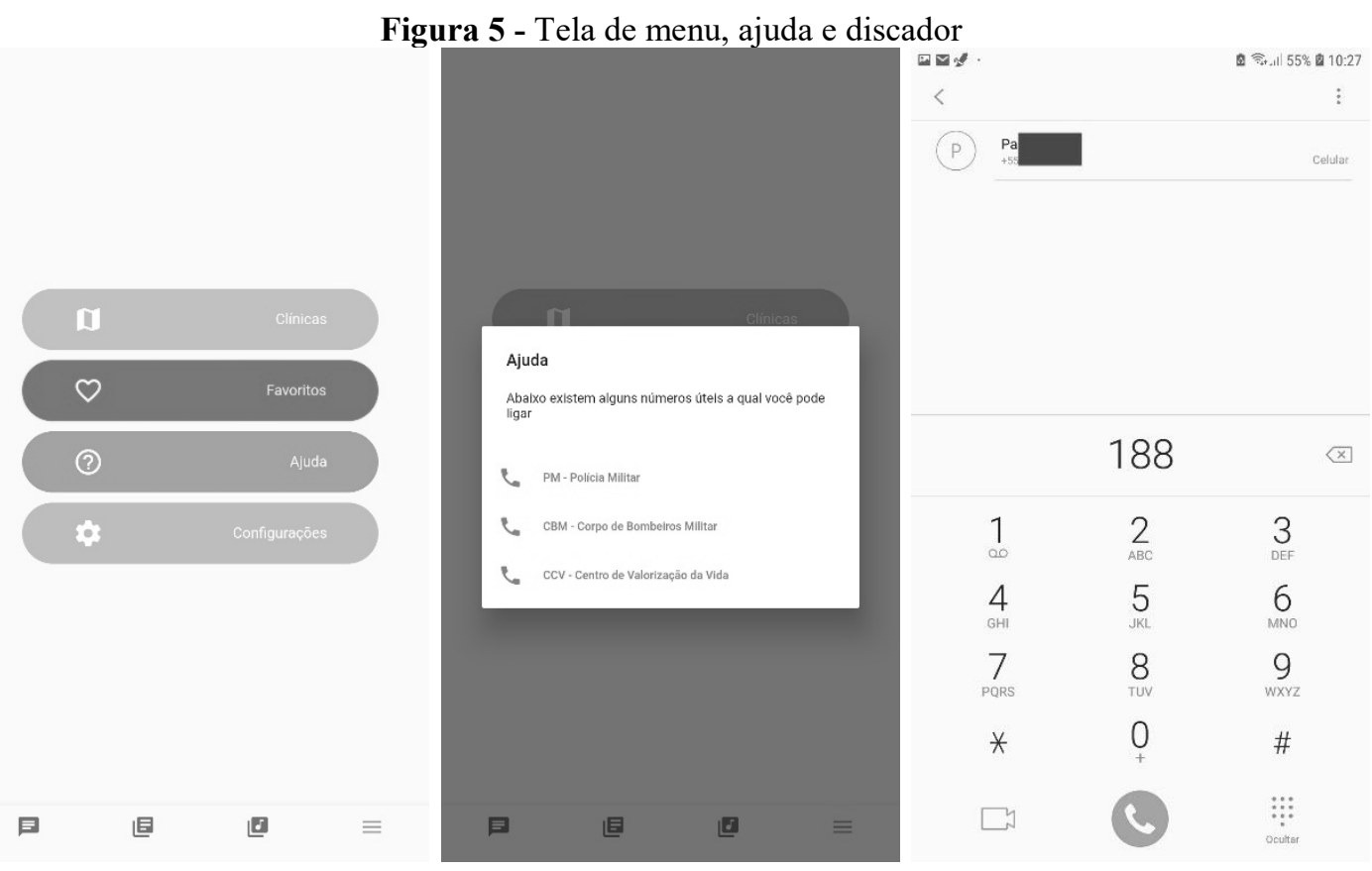

Fonte: O Autor 


\section{Conclusão}

O desenvolvimento desta ferramenta pode não só melhorar a qualidade de vida das pessoas como facilitar a análise e diagnóstico por parte do profissional da saúde. Durante o desenvolvimento do projeto, por ser um tema bastante delicado, obteve-se ajuda de profissionais formados para que nada fosse feito sem o respaldo de uma pessoa que realmente entendesse do assunto.

O aplicativo encontra-se parcialmente pronto, ou seja, pode ser utilizado perfeitamente com as funcionalidades que já existem, porém, o projeto não está concluído. Algumas características citadas e não citadas estão na etapa final de desenvolvimento. O projeto segue em aberto para implementação de algumas "features" como:

1- Rede Social entre profissionais e usuários, sendo eles pacientes ou não.

2- Gráfico de estado mental do usuário

3- Músicas, playlists calmas e relaxantes e podcasts informativos.

Deu-se início a fase de testes e coleta de feedback dos usuários afim de proporcionar uma melhora significativa na qualidade e tempo de desenvolvimento do projeto.

\section{Referências}

${ }^{1}$ Anatel [homepage na internet]. Relatório Analítico 2018 [acesso em 04 Jul 2020]. Disponível em: https://www.anatel.gov.br/publicacoes/pesquisa_relatorio_analitico_2018_final.pdf

${ }^{2}$ National Health System - UK [homepage na internet]. Clinical depression - Causes [acesso em 04 Jul 2020]. Disponível em: https://www.nhs.uk/conditions/clinicaldepression/causes/

${ }^{3}$ Ministério da Saúde [homepage na internet]. Depressão: causas, sintomas, tratamentos, diagnóstico e depressão [acesso em 04 Jul 2020]. Disponível em: http://portalms.saude.gov.br/saude-de-a-z/saude-mental/depressao

${ }^{4}$ Vittude [homepage na internet]. Depressão: causas, sintomas físicos, tratamentos e prevenção [acesso em 04 Jul 2020]. Disponível em: https://www.vittude.com/blog/depressao/

${ }^{5}$ Enferm. vol.21 no.2 Florianópolis Apr./June 2012 [homepage na internet]. Tecnologia, inovação tecnológica e saúde: uma reflexão necessária [acesso em 04 Jul 2020]. Disponível em: https:/www.scielo.br/scielo.php?pid=S010407072012000200023\&script=sci_arttext

6 Vazquez, Carlos; Simões, Guilherme (2016). Engenharia de Requisitos: Software Orientado ao Negócio. [S.1.]: Brasport

${ }^{7}$ National Institute of Mental Health [homepage na internet]. Depression: What You Need To Know [acesso em 04 Jul 2020]. Disponível em: https://www.nimh.nih.gov/health/publications/depression-what-you-need-toknow/index.shtml

z/depressao\#: :text=A\%20depress $\% \mathrm{C3} \% \mathrm{~A} 3 \mathrm{o} \% 20 \% \mathrm{C3} \% \mathrm{~A} 9 \% 20 \mathrm{um} \% 20$ problema,torno $\% 20 \mathrm{de} \% 2015 \% 2 \mathrm{C} 5 \% 25$. 